Editorial

\title{
Updates on peptide vaccines
}

Volume I Issue I - 2015

\begin{abstract}
Abbreviations: HER, heregulin; CEA, carcinoembryonic antigen; MUC-1, mucin-1; PSMA, prostate-specific membrane antigen; MAP, multiple antigenic peptide; GnRH, gonadotropin releasing hormone; hCG, human chorionic gonadotropin; $\mathrm{ZP}$, zona pellucid; FA, fertilization antigen; RSA, rabbit sperm autoantigen
\end{abstract}

\section{Editorial}

Current existing vaccines are synthesized from various inactivated disease causing organism or attenuated version of pathogen or else suitable part of it e.g. toxin. However the response from the immune system to the antigen is with respect to small number of amino acid residues or peptide. Synthetic peptide vaccines characterize protein antigen fragments, which are produced and assembled from amino acid molecules to a supramolecular complex, predicted by immune system and causes immune response. This immune response is induced either by B-cells or T-cells as well as involves both possible pathways. So the chief component of peptide vaccines are the protein fragments exhibiting $\mathrm{T}$ and/or $\mathrm{B}$ epitope function, which regulates the specificity and direction of the immune responses. These synthetic peptide vaccines may also enclose some distinct complexes or supramolecular structures like polymer particles, liposomes, micelles, etc can specifically or nonspecifically trigger our immune system immune to synthetic fragments and thereby initiating the immune response. ${ }^{1}$ The structural and proteolytic stability of the synthetic peptides is further improved by linking to genetically programmed cross-linking carriers that subsequently act as activators for the immune system to respond. ${ }^{2}$

Numerous vaccines based upon several peptides sequences have been tried for cancer immunotherapy. The notable peptide vaccines that have undergone phase I/II/III clinical trials are Heregulin (HER)2/neu peptide, Carcinoembryonic antigen (CEA), Mucin-1 (MUC1) peptide, Prostate-specific membrane antigen (PSMA), ALVACCEA vaccine, Ras oncoprotein, Melanoma antigens IFN, peptidegranulocyte-macrophage colony-stimulating factor, GP2 vaccine, AE37 vaccines, E75 vaccine, GPC3-derived peptide vaccine, GV1001 Dendritic Cells pulsed with four AFP peptides modified DNA aptamer TLS11a-GC, anti-OX40 monoclonal antibody with two folate binding protein peptide, Vaccines (E39 and J65), Bombesin/ Gastrin-releasing peptide. Peptide vaccines for have been tried for various infectious diseases such as licensed HPV peptide-based vaccine contains Montanide and Multiple antigenic peptide (MAP). Peptide therapeutics in treating type 2 diabetes includes CBX129801, CVX060, LAPSExd4, PB1023, IICBP501, AM111 and ACT1. The following peptide vaccines based upon reproductive hormones and gamete antigens have been investigated for immunoregulation of fertility such as Gonadotropin releasing hormone $(\mathrm{GnRH})$ peptide, Human chorionic gonadotropin (hCG) peptide, Oocyte zona pellucida (ZP), Fertilization antigen (FA)-1 peptide, YLP12 peptide, Rabbit sperm autoantigen (RSA)/Sp17 peptide, Sp56 peptide, YWKII peptide, rSM9P-B peptide, LDH-C4 peptide and Multi-sperm peptides combination vaccine (YLP12, P10G, A9D, mFA-12-19, SP56 and mFA-1117-136).

Commercially available peptide drugs have been successfully applied in treating certain human diseases such as Goserelin (a

\section{Shilu Mathew,' Ishtiaq Qadri}

'Center of Excellence in Genomic Medicine, King Abdul Aziz University, Saudi Arabia

${ }^{2}$ King Fahd Medical Research Center, King Abdul Aziz University, Saudi Arabia

Correspondence: Ishtiaq Qadri, Medical Biotechnology and Translational Medicine Research, King Fahd Medical Research Center, King Abdul Aziz University, PO Box 80216 Jeddah 21589, Saudi Arabia, Email ishtiaq80262@yahoo.com

Received: March 29, 2015 | Published: April 0I, 2015

synthetic gonadotropin-releasing hormone analog, marketed as Zoladex) is applied to treating breast cancer and prostate cancer. Glatiramer acetate (a synthetic peptide with four amino acids, Copaxone) is used for multiple sclerosis and Exenatide (a synthetic glucagon-like peptide-1 analog, Byetta) for type 2 diabetes. The synthetic somatostatin analogs such as octreotide (Sandostatin) and lanreotide (Somatuline) are the most common drugs used in treating neuroendocrine. In the commercial market, synthetic peptide drugs generating more than \$1billion in annual sales, such as Lupron, Zoladex, Copaxone and Sandostatin. ${ }^{3}$ However, the approval rate for peptide drugs may be twice as high as that for small molecules. ${ }^{4}$ The peptide drug market is also growing twice as fast in the worldwide drug market. ${ }^{5}$ Currently, there are around 60-70 approved peptide drugs in the global market, with 100-200 more in clinical trials, 400600 more in pre-clinical studies ${ }^{4,5}$ and possibly hundreds to thousands more on the laboratory bench.

As more pathogen genomes are revealed and our understanding of fundamental immunological mechanisms deepens, it becomes ever clearer that the only tractable approach to vaccine discovery and design is through the interplay of in silico with in vitro and in vivo techniques. Indeed, the combination of computational and experimental epitope mapping offers many tools and techniques appropriate to the identification and optimization of vaccine candidates. Variety of approaches will fully synergize to speed up the development of powerfully efficacious vaccines against both infective and chronic disease states. With the barriers having been broken, it will be open season for peptide drugs and their hunters for the future.

\section{Acknowledgments}

None.

\section{Conflicts of interest}

Author declares there are no conflicts of interest. 


\section{Funding}

None.

\section{References}

1. Vogel FR, Carl R, Alving. Progress in immunologic adjuvant development: 1982-2002. In: Heilman C, et al. (Eds.), Accelerated Development of Vaccines. The Jordan Report, NIH/NIAID, USA. 2002. p.39-43.

2. Aguilar JC, Rodrigue EG. Vaccine Adjuvants Revisited. Vaccine. 2007;25(19):3752-3762.
3. Reichert J. Development Trends for Peptide Therapeutics (2010 Report Summary). Peptide Therapeutics Foundation. 2010. p.1-10.

4. Lax R. The Future of Peptide Development in the Pharmaceutical Industry. PharManufacturing. 2010. p.10-15.

5. Bellmann-Sickert K, Annette G, Beck-Sickinger. Peptide drugs to target $\mathrm{G}$ protein-coupled receptors. Trends in Pharmacological Sciences. 2010;31(9):434-441. 